\title{
PHƯƠNG PHÁP NÂNG CAO NĂNG LỰC CỦA GIẢNG VIÊN ĐỂ TRIỂN KHAI TỐT CÔNG TÁC ĐÀO TẠO THEO HƯỚNG TIẾP CẬN CDIO Nguyễn Thị Hiền ${ }^{(*)}$, Nguyễn Thị Huệ $\hat{e}^{(*)}$
}

(*) Thạc sĩ. Truòng Đại học Công nghệĐồng Nai. Email: nguyenthihien@dntu.edu.vn

DOI: $10.37550 /$ tdmu.CFR/2021.01.136

\section{Tóm tắt}

Bài viết này đề cấp đến cách thực hiện để nâng cao năng lực về kỹ năng, giảng dạy cho giảng viên tại trường Đại học Công nghệ Đồng Nai nhằm mục đích thục hiện tốt việc đào tạo theo định hương CDIO.Truò̀ng đại học có các ngành khối kỹ thuật chiếm trên 50\% tổng số các ngành đào tạo nên việc triển khai công tác đào tạo theo huớng tiếp cận CDIO đã được nhà trường chú trọng phát triển trong nhiều năm gần đây. Tù việc mời chuyên gia nước ngoài có nhiều năm kinh nghiệm giảng dạy theo phưong pháp CDIO về tập huấn cho đội ngũ giảng viên cách cập nhật chuơng trình đào tạo theo phuơng pháp CDIO, cách triển khai một học phần theo phuơng pháp CDIO đến việc mời chuyên gia trong nước đến tập huấn mô hình giảng dạy CDIO, tập huấn xây dựng chuẩn đầu ra chương trình đào tạo theo mô hình CDIO... Tất cả đã thu được các kết quả đáng mong đợi. Tù kết quả này, chúng tôi muốn chia sẻ cách làm của Trường để góp phần nâng cao hơn nữa tinh thần đoàn kêt và sự hợp tác trên mọi phương diện.

Tù̀ khóa: mô hình CDIO, kết quả đầu ra, dụ án

\section{1. Đặt vấn đề}

Sáng kiến CDIO ra đời như một giải pháp điển hình cho giáo dục đại học nhằm bắt kịp xu thế là Đào tạo đáp ứng nhu cầu xã hội và hội nhập quốc tế. Tầm nhìn của dự án là mang đến cho sinh viên một nền giáo dục nhấn mạnh về nền tảng kỹ thuật trong bối cảnh Hình thành ý tưởng - Thiết kế - Triển khai - Vận hành (Conceiving - Designing - Implementing Operating - CDIO) các hệ thống và sản phẩm thực tế. Quy trình này được xây dựng một cách khoa học, hợp lý, logic, có thể áp dụng cho nhiều lĩnh vực đào tạo khác nhau. Nhằm giúp người học đạt được ba mục tiêu chung đó là: Nắm vững kiến thức chuyên sâu của nền tảng kỹ thuật. Dẫn đầu trong việc kiến tạo và vận hành sản phẩm vào hệ thống mới. Hiểu được tầm quan trọng và tác động chiến lược của nghiên cứu và phát triển công nghệ đối với xã hội. Để đồng bộ hóa sản phẩm đầu ra của giáo dục, nhà trường đã từng bước đào tạo, bồi dưỡng nâng cao trình độ và năng lực giảng viên bằng những hoạt động thiết thực. 


\section{Tập huấn nâng cao năng lực giảng viên}

\subsection{Tập huấn các nội dung liên quan đến CDIO}

Nhận thấy, hướng tiếp cận CDIO là xu thế tất yếu nên nhà trường đã triển khai kế hoạch đào tạo giảng dạy theo định hướng $\mathrm{CDIO}$ cho tất cả các giảng viên trong trường thông qua chương trình hợp tác với tổ chức phi chính phủ PUM - Hà Lan từ tháng 2 năm 2016 đến tháng 3 năm 2018 (kế hoạch như bảng 2 trong phụ lục kèm hình ảnh như hình 1). Tiếp đến là mời chuyên gia về CDIO từ Trường Đại học khoa học tự nhiên TPHCM đến tập huấn và cấp chứng chỉ (hình 2 , hình 3 , hình 4 phần phụ lục). Nội dung tập huấn về CDIO bao gồm:

- Xây dựng chuẩn đầu ra (CĐR) theo CDIO

- Xây dựng khung chương trình đào tạo tích hợp các kỹ năng cá nhân, kỹ năng giao tiếp; kỹ năng kiến tạo sản phẩm, qui trình, hệ thống; kỹ năng và thái độ chuyên nghiệp; đạo đức nghề nghiệp; ... nhằm đảm bảo sinh viên đạt được $\mathrm{C} Đ R$ theo $\mathrm{CDIO}$.

• Các phương pháp dạy, học chủ động và trải nghiệm.

- Phương pháp đánh giá sinh viên cho lớp ít và lớp đông (hỗ trợ xây dựng Rubric, xác định tiêu chí và tiêu chuẩn).

• Thiết kế đề cương môn học, triển khai học phần theo hướng CDIO.

• Xây dựng kế hoạch bài giảng với việc kết hợp các phương pháp giảng dạy chủ động và phương pháp đánh giá tiên tiến.

Số lượng giảng viên tham gia tập huấn ứng với mỗi nội dung là khác nhau tùy theo đặc trưng công việc, chức vụ và ngành nghề. Sau mỗi nội dung tập huấn giảng viên đều được giao hoàn thành một nội dung công việc một cách tương ứng và là cơ sở để cấp giấy chứng nhận. Sau 3 năm triển khai, nhà trường đã thu được một số thành quả sau:

- Xác định được chuẩn đầu ra là gì, xây dựng được chuẩn đầu ra theo CDIO đảm bảo các cấp bậc đồng thời đảm bảo chiều sâu và rộng nhằm định hướng được sinh viên ra trường đáp ứng được với nhu cầu xã hội ngày càng đòi hỏi cao. Chuẩn đầu ra có sự tương đồng và có đặc trưng riêng của mỗi ngành.

- Cập nhật chương trình đào tạo mới sao cho giảm thời lượng lý thuyết, tăng thực hành, thí nghiệm; sinh viên sẽ được học tập theo hướng tích hợp liên môn chứ không chỉ đơn môn như trước đây, đồng thời bỏ những học phần không còn phù hợp với thực tế hiện nay, thêm những học phần có tính đáp ứng nhu cầu xã hội. Ngoài ra còn chú trọng đến các kỹ năng mềm cho sinh viên và trình độ ngoại ngữ trong thời kỳ hội nhập quốc tế.

• Giảng viên thiết kế được đề cương chi tiết học phần có sự gắn kết với giáo trình, tài liệu tham khảo. Trong đó thể hiện cụ thể hình thức đánh giá học phần, thang điểm cùng với rucric đánh giá đi kèm. Ngoài ra còn thể hiện phương pháp giảng dạy và học tập kết hợp, chủ động để đạt được mục tiêu học phần - gắn kết với chuẩn đầu ra chương trình.

- Giảng viên xây dựng được kế hoạch giảng dạy trên hệ thống để sinh viên nhìn thấy như một bản kế hoạch giúp sinh viên chủ động hơn trong việc học. 


\subsection{Tập huấn xây dụng bài giảng Elearning}

Trường có một hệ thống Elearning riêng và giảng viên được tập huấn bài bản không những về cách sử dụng Elearning mà còn được tập huấn về các công cụ tạo các cuộc họp trực tuyến như Cisco Webex hay Microsoft Team. Cuối khóa tập huấn, giảng viên được thực hành làm một học phần trên hệ thống Elearning với đầy đủ các yêu cầu để bộ phận phụ trách đánh giá mức độ hoàn thành. Ngoài ra, mỗi Khoa sẽ thành lập một bộ phận thẩm định hồ sơ giảng dạy Elearning của giảng viên. Giảng viên đăng ký dạy online sẽ được thẩm định hồ sơ giảng dạy trước khi thực hiện. Hồ sơ Elearning chưa đạt yêu cầu sẽ không được giảng dạy online. Chính vì nắm bắt được xu thế này mà nhà trường vẫn duy trì được hoạt động dạy và học trong hai đợt dịch Covit 19 nghiêm trọng vừa qua.

Bài giảng Elearning là một hồ sơ giảng viên phải chuẩn bị đầy đủ trước lịch dạy. Đây cũng là một kênh thông tin chính góp phần tăng tính chủ động học, nghiên cứu và chủ động trao đổi của sinh viên.

Trong hồ sơ giảng viên trên Elearning thể hiện rõ: Đề cương chi tiết học phần; mức độ đáp ứng mục tiêu, chuẩn đầu ra; kế hoạch giảng dạy học phần; hoạt động tương tác; hình thức kiểm tra, đánh giá; nguồn học liệu. Đây được coi như một chuẩn hồ sơ giảng viên cung cấp cho người học những thông tin quan trọng giúp họ định hướng tốt hơn, giao tiếp tốt hơn với người dạy và người học để đạt được chuẩn đầu ra như mong đợi.

\subsection{Tập huấn phần mềm Canvas}

Cuộc cách mạng khoa học công nghệ diễn ra mạnh mẽ trong thời gian qua đã tạo ra nhiều sản phẩm công nghệ, phần mềm với nhiều tính năng hơn và được ứng dụng rộng rãi vào nhiều lĩnh vực đời sống, xã hội, làm thay đổi xu thế, phương thức hoạt động của một ngành nghề, lĩnh vực, trong đó có giáo dục. Với sự hỗ trợ của các trang thiết bị, phần mềm hiện nay đã tạo điều kiện thuận lợi để các trường đại học, sinh viên có điều kiện thuận lợi để triển khai thực hiện giảng dạy, học tập online nhằm phù hợp với xu thế của xã hội trong bối cảnh hội nhập quốc tế, trong đó nổi bật là phần mền Canvas ứng dụng trong lĩnh vực giáo dục.

Trường Đại học Công nghệ Đồng Nai đã trang bị phần mềm Canvas có tích hợp nhiều chức năng hơn như tạo Trang, tạo Mô đun, quay phim, chèn câu hỏi trắc nghiệm trên video...Tất cả những chức năng đó giúp cho người dạy thỏa sức sáng tạo trong thiết kế bài giảng nhằm tạo hứng thú, gây sự chú ý và đặc biệt hơn là truyền cảm hứng tốt hơn đến người học.

Bắt đầu tham gia là giảng viên khoa ngoại ngữ được tập huấn từ các chuyên gia nước ngoài (từ 12/7/2020 đến 15/8/2020). Song song với thời gian tập huấn online đó thì giảng viên khoa ngoại ngữ thực hiện bài giảng từng phần và quay video tại trường quay DNTU. Mỗi giảng viên khoa ngoại ngữ được phân công phụ trách một nội dung về Canvas. Giảng viên vừa đóng vai người học, vừa đóng vai người dạy. Sau thời gian hơn một tháng, đội ngũ giảng viên khoa ngoại ngữ tiến hành tập huấn cho tất cả các giảng viên còn lại (từ 17/8/2020 đến 25/8/2020) và kết thúc khóa tập huấn, giảng viên còn lại được yêu cầu làm một bài thi trên Canvas với đầy đủ các nội dung được tập huấn. Bài thi gồm phần trắc nghiệm chiếm tỉ trong $10 \%$ và phần thiết kế bài giảng chiếm tỉ trọng $90 \%$. Giảng viên hoàn thành bài thi là đạt điểm từ 7 điểm trở lên được 
cấp chứng chỉ hoàn thành khóa tập huấn Canvas và được giảng dạy trên hệ thống này. Kết quả thi Canvas như bảng 1 sau:

Bảng 1. Tỉ lẹ giảng viên (GV) thi Canvas

\begin{tabular}{|c|c|c|c|c|}
\hline $\begin{array}{c}\text { Tổng số GV tham } \\
\text { gia }\end{array}$ & Số GV đạt & Số GV không đạt & Tỉ lệ GV đạt & Tỉ lệ GV đạt \\
\hline 116 & 102 & 14 & $88 \%$ & $12 \%$ \\
\hline
\end{tabular}

Trong thời đại bùng nổ của khoa học công nghệ thì người học và người dạy có thể giao tiếp được với nhau mọi lúc mọi nơi. Cả người dạy và người học chủ động hơn trong việc dạy và học đặc biệt là theo trình tự: Hình thành ý tưởng - thiết kế - triển khai - vận hành. Theo quy trình này, thông qua giao tiếp thì mỗi bên tự tìm cho mình cách giải quyết vấn đề sao cho đạt hiệu quả cao nhất và đạt được chuẩn đầu ra.

\subsection{Tổ chức thi nghiệp vụ cố vấn học tập}

Song song với việc trang bị cho giảng viên các kiến thức, kỹ năng mới về công nghệ để đảm bảo dạy theo dự án mọi lúc mọi nơi, bắt kịp và hòa nhập với xu thế thì một yếu tố không kém phần quan trọng là sự giao tiếp thường xuyên giữa người dạy và người học. Hay hiểu đơn giản là có sự quan tâm, chăm sóc tới người học thông qua việc tư vấn các quy định, nội quy của nhà trường tới người học, những việc mà người học được làm và không được làm, những quyền lợi mà người học được hưởng ... Những thông tin đó, người dạy phải nắm rõ để hỗ trợ sinh viên được tốt nhất có thể. Nên nhà trường đã tổ chức thi trắc nghiệm công tác cố vấn học tập với ngân hàng đề thi 300 câu. Đề thi ngẫu nhiên gồm 70 câu cho một giảng viên. Kết quả thi phải đạt từ 8.5 điểm trở lên. Những giảng viên chưa đạt yêu cầu đều phải thi lại.

\subsection{Bồi duỡng $k \tilde{y}$ năng mềm}

Giảng viên cũng được bồi dưỡng các kỹ năng mềm để phục vụ tốt hơn cho việc giảng dạy như kỹ năng thuyết trình, kỹ năng làm việc nhóm, kỹ năng giải quyết vấn đề... Lớp bồi dưỡng này do phòng quan hệ doanh nghiệp và phát triển kỹ năng phụ trách. Giảng viên được bồi dưỡng bằng cách tham gia một lớp học gồm 30 đến 50 giảng viên có thời lượng 60 tiết. Giảng viên hoàn thành lớp bồi dưỡng được cấp chứng nhận đã tham gia lớp bồi dưỡng kỹ năng. Đây cũng là dịp giảng viên ôn lại những hoạt động trong giao tiếp giảng dạy để truyền đạt kiến thức, kỹ năng và nhiệt huyết tới người học được tốt nhất.

\section{Kết quả đạt được}

Để nâng cao chất lượng giảng dạy, giảng viên phải được đào tạo hoặc tự đào tạo mình để đào tạo sinh viên sau khi tốt nghiệp có thể tự hòa nhập được với môi trường làm việc, gắn kết với xã hội và bắt kịp với thời đại. Sau một thời gian triển khai các hoạt động nâng cao năng lực giảng viên, Nhà trường đã gặt hái được những thành công nhất định.

Tính đến nay có 17/18 ngành đào tạo của Trường áp dụng định hướng CDIO trong đó có 10 ngành thuộc khối kỹ thuật. Ở mỗi ngành, tùy theo đặc thù riêng mà có học phần áp 
dụng 100\% CDIO hoặc chỉ áp dụng một phần hay kết hợp để phù hợp với tính chất chuyên môn và với khả năng của người học nhằm đảm bảo chuẩn đầu ra. Những học phần áp dụng giảng dạy theo dự án có tỉ lệ sinh viên đậu trên $96 \%$. Sinh viên không qua kỳ thi là do đi làm thêm nhiều hoặc trốn nghĩa vụ quân sự.

Với sự nỗ lực không ngừng của đội ngũ lãnh đạo và toàn thể cán bộ, nhân viên, giảng viên của Trường, cùng với sự góp sức không nhỏ của sinh viên, các nhà tuyển dụng, các chuyên gia được mời về. Trường Đại học Công nghệ Đồng Nai không ngừng nâng cao chất lượng đào tạo, đưa một trường chưa hề có tên tuổi đến trường đạt kiểm định chất lượng giáo dục năm 2017 với mức đánh giá 83,5\%. Đầu năm 2019, Trường kiểm định thành công 2 ngành với mức đánh giá $86 \%$. Không dừng lại đó, trong năm 2020, Trường tiếp tục được kiểm định 6 ngành đào tạo khác. Và trong báo cáo của đoàn đánh giá ngoài luôn dành cho trường lời khen về tỉ lệ sinh viên có việc làm sau khi tốt nghiệp 1 năm là $95 \%$, một con số đáng ngưỡng mộ.

\section{Kết luận}

Giảng dạy theo hướng tiếp cận CDIO hay gọi ngắn gọn là dạy học theo dự án đòi hỏi cả người dạy và người học đều phải nỗ lực không ngừng. Trường Đại học Công nghệ Đồng Nai đã thực hiện thành công đề án đổi mới hoạt động giai đoạn 2016 - 2020 và đã liên tục học tập, áp dụng và triển khai mô hình CDIO trong đào tạo. Đây là quá trình thay đổi phương pháp giảng dạy, phương pháp đánh giá để đảm bảo sinh viên là đối tượng được thụ hưởng kiến thức một cách mạch lạc sáng tạo nhất. Từ đó cho thấy: Việc xây dựng chuẩn đầu ra, chương trình đào tạo, đề cương theo mô hình CDIO là yếu tố cốt lõi và cần thiết để tạo ra sản phẩm lao động với kiến thức, kỹ năng và thái độ nghề nghiệp đáp ứng yêu cầu thực tế. Nhà Trường không ngừng học tập kinh nghiệm từ các trường Đại học đi trước để đạt nhiều kết quả tốt trong việc triển khai mô hình giảng dạy CDIO. Kết quả là Trường đã gia nhập Hiệp hội CDIO khu vực Châu Á.

\section{Tài liệu tham khảo}

[1] Tập huấn nâng cao năng lực giảng viên để giảng dạy chương trình CDIO, Phùng Thúy Phượng, Phan Nguyễn Ái Nhi, Lê Mỹ Loan Phụng, Nguyễn Thị Huyền, Đồng Thị Bích Thủy, Trung Tâm Cải Tiến Phương Pháp Dạy và Học Đại Học (CEE), Trường Đại Học KHTN - ĐHQG TPHCM, 2012.

[2] "Kỷ yếu Hội thảo Quốc tế về CDIO”, Đại học Quốc gia TP Hồ Chí Minh, 13-14/12/2010

[3] Hồ Tấn Nhựt, “The CDIO approach to engineering education: Introduction”, 2008. 


\section{PHỤ LỤC}

Bảng 2. Trích lịch làm việc của chuyên gia PUM tù̀ tháng 2 năm 2016 đến tháng 3 năm 2018

\begin{tabular}{|c|c|c|c|c|c|}
\hline Ngày & Giờ & Nội dung & Chủ trì & Địa điểm & Ghi chú \\
\hline \multirow[t]{2}{*}{$\begin{array}{c}\text { Thứ } 2 \\
\text { 29-02-2016 }\end{array}$} & $08 \mathrm{~h} 00$ & $\begin{array}{l}\text { Gặp gỡ và làm việc với chuyên gia PUM - } \\
\text { Ms. Anneke Bal- Atsma. } \\
\text { Thành phần: Trưởng/ phó thường trực các } \\
\text { khoa chuyên ngành - Thầy Phạm Sắc, Thầy } \\
\text { Lưu Hồng Quân, Thầy Trần Thanh Đại, } \\
\text { Thầy Nguyền Hoàng Hưng, Thầy Vũ Thịnh } \\
\text { Trường, Cô Phạm Thị Hải Vân }\end{array}$ & $\begin{array}{l}\text { PHT - TS Trần } \\
\text { Đức Thuận }\end{array}$ & $\begin{array}{l}\text { Phòng họp } \\
4\end{array}$ & $\begin{array}{l}\text { - Phòng HCTH } \\
\text { chuẩn bì máy } \\
\text { chiếu, màn chiếu, } \\
\text { amplifier, } 2 \text { micro } \\
\text { (pin) } \\
\text { - Phòng QHDN } \\
\text { chụp hình \& đưa } \\
\text { tin }\end{array}$ \\
\hline & $13 \mathrm{~h} 30$ & PUM làm việc với Khoa Ngoại ngữ & $\begin{array}{l}\text { PHT - TS Trần } \\
\text { Đức Thuận }\end{array}$ & $\begin{array}{l}\text { Phòng họp } \\
4\end{array}$ & \\
\hline \multirow{2}{*}{$\begin{array}{c}\text { Thứ } 3 \\
01-03-2016\end{array}$} & 08h00 & $\begin{array}{l}\text { PUM làm việc với Khoa Quản trị \& Khoa } \\
\text { Kế toán Tài chính } \\
\text { Thành phần: Phó Trưởng Khoa, Tổ trưởng } \\
\text { bộ môn, giảng viên không có giờ giảng }\end{array}$ & $\begin{array}{l}\text { PHT }- \text { TS. } \\
\text { Trần } \\
\text { Thuận }\end{array}$ & $\begin{array}{l}\text { Phòng họp } \\
4\end{array}$ & \\
\hline & $13 \mathrm{~h} 30$ & $\begin{array}{l}\text { PUM làm việc với Khoa Đ, ĐT - CK \&XD } \\
\text { Thành phần: Phó Trưởng Khoa, Tổ trưởng } \\
\text { bộ môn, giảng viên không có giờ giảng }\end{array}$ & $\begin{array}{l}\text { PHT }- \text { TS. } \\
\text { Trần } \\
\text { Thuận }\end{array}$ & $\begin{array}{l}\text { Văn phòng } \\
\text { Khoa } \\
\text { Đ,ĐT- } \\
\text { CK\&XD }\end{array}$ & \\
\hline \multirow{2}{*}{$\begin{array}{c}\text { Thứ } 4 \\
02-03-2016\end{array}$} & $08 \mathrm{~h} 00$ & $\begin{array}{l}\text { PUM làm việc với khoa TP-MT \& ĐD } \\
\text { Thành phần: Phó trưởng Khoa, Tổ trưởng } \\
\text { bộ môn, giảng viên không có giờ giảng }\end{array}$ & $\begin{array}{l}\text { PHT }- \text { TS. } \\
\text { Trần } \\
\text { Thuận }\end{array}$ & $\begin{array}{l}\text { Văn phòng } \\
\text { Khoa TP- } \\
\text { MT\&ĐD }\end{array}$ & \\
\hline & $13 \mathrm{~h} 30$ & $\begin{array}{l}\text { PUM làm việc với Khoa Công nghệ thông } \\
\text { tin } \\
\text { Thành phần: Phó trưởng khoa, Tổ trưởng bộ } \\
\text { môn, giảng viên không có giờ giảng }\end{array}$ & $\begin{array}{l}\text { PHT }- \text { TS. } \\
\text { Trần } \\
\text { Thuận }\end{array}$ & $\begin{array}{l}\text { Phòng họp } \\
4\end{array}$ & \\
\hline $\begin{array}{c}\text { Thứ } 5 \\
03-03-2016\end{array}$ & $08 \mathrm{~h} 00$ & $\begin{array}{l}\text { PUM báo cáo thuyết trình kế hoạch hoạt } \\
\text { động } \\
\text { Thành phần: Trưởng, Phó thường trực các } \\
\text { Khoa chuyên }\end{array}$ & $\begin{array}{l}\text { PHT }- \text { TS. } \\
\text { Trần } \\
\text { Thuận }\end{array}$ & $\begin{array}{l}\text { Phòng họp } \\
4\end{array}$ & \\
\hline $\begin{array}{c}\text { Thứ } 6 \\
04-03-2016\end{array}$ & $09 \mathrm{~h} 00$ & $\begin{array}{l}\text { Họp mặt } 3 \text { bên: PUM, Tổng Công ty dệt } \\
\text { may Đồng Nai \& DNTU } \\
\text { Thành phần: Phó trưởng Phòng QHDN, đại } \\
\text { diện Tổng Công ty dệt may Đồng Nai, đại } \\
\text { diện PUM tại Việt Nam: bà Hoàng Lan, } \\
\text { chuyên gia PUM }\end{array}$ & $\begin{array}{l}\text { PHT }- \text { TS. } \\
\text { Trần } \\
\text { Thuận }\end{array}$ & $\begin{array}{l}\text { Phòng họp } \\
2\end{array}$ & \\
\hline $\begin{array}{c}\text { Thứ } 2 \\
07-03-2016\end{array}$ & $\begin{array}{l}08 \mathrm{~h} 00- \\
11 \mathrm{~h} 00\end{array}$ & $\begin{array}{l}\text { Tiết dạy mẫu của chuyên gia PUM: tương } \\
\text { tác điện thoại di động qua wifi vào bài } \\
\text { giảng } \\
\text { Thành phần: giảng viên khoa Đ,ĐT-CK } \\
\text { \&XD không có giờ dạy }\end{array}$ & $\begin{array}{l}\text { PHT - TS. Trần } \\
\text { Đức Thuận }\end{array}$ & $\begin{array}{l}\text { Văn phòng } \\
\text { Khoa Điện, } \\
\text { Điện tứ - } \\
\text { Cơ khí và } \\
\text { Xây dựng }\end{array}$ & \\
\hline Thứ 5 & 09h15- & Chuyên gia PUM trao đổi với Giảng viên, & PHT - TS. Trần & Phòng họp & Phòng QHDN cử \\
\hline
\end{tabular}




\begin{tabular}{|c|c|c|c|c|c|}
\hline $10-03-2016$ & $10 \mathrm{~h} 00$ & $\begin{array}{l}\text { sinh viên về phản hồi của Doanh nghiệp đối } \\
\text { với công tác tồ chức thực tập cho sinh viên } \\
\text { Thành phần: Giảng viên các Khoa không có } \\
\text { giờ dạy, sinh viên các Khoa }\end{array}$ & Đức Thuận & 3 & $\begin{array}{l}\text { NV chụp hình, } \\
\text { đăng tin }\end{array}$ \\
\hline $\begin{array}{c}\text { Thứ } \mathbf{6} \\
\text { 11-03-2016 }\end{array}$ & $\begin{array}{l}08 \mathrm{~h} 00- \\
11 \mathrm{~h} 00\end{array}$ & $\begin{array}{l}\text { Báo cáo, tổng kết của PUM } \\
\text { Thành phần tham dự: Chuyên gia PUM, } \\
\text { Trưởng, Phó các Khoa }\end{array}$ & $\begin{array}{l}\text { PHT - TS. Trần } \\
\text { Đức Thuận }\end{array}$ & $\begin{array}{l}\text { Phòng họp } \\
4\end{array}$ & $\begin{array}{l}\text { Phòng QHDN cử } \\
\text { NV chụp hình, } \\
\text { đăng tin }\end{array}$ \\
\hline $\begin{array}{c}\text { Thứ } \mathbf{6} \\
05-08-2016\end{array}$ & $07 \mathrm{~h} 30$ & $\begin{array}{l}\text { Tổ chức khóa học "Nâng cao phương pháp } \\
\text { giảng dạy giúp sinh viên học tập chủ động } \\
\text { và trải nghiệm, đạt các chuẩn đầu ra theo } \\
\text { CDIO" chuyên đề } 1 \\
\text { Thành phần: Cán bộ, giảng viên đã đăng ký } \\
\text { theo lịch }\end{array}$ & $\begin{array}{l}\text { PHT - TS. Trần } \\
\text { Đức Thuận }\end{array}$ & $\begin{array}{l}\text { Phòng họp } \\
3\end{array}$ & \\
\hline \multirow{2}{*}{$\begin{array}{c}\text { Thứ } 2 \\
22-08-2016\end{array}$} & 07h30 & $\begin{array}{l}\text { Tổ chức khóa học "Nâng cao phương pháp } \\
\text { giảng dạy giúp sinh viên học tập chủ động } \\
\text { và trải nghiệm, đạt các chuẩn đầu ra theo } \\
\text { CDIO" chuyên đề } 2 \\
\text { Thành phần: Cán bộ, giảng viên đã đăng ký } \\
\text { theo lịch }\end{array}$ & $\begin{array}{l}\text { PHT - TS. Trần } \\
\text { Đức Thuận }\end{array}$ & $\begin{array}{l}\text { Phòng họp } \\
3\end{array}$ & \\
\hline & $13 \mathrm{~h} 30$ & $\begin{array}{l}\text { Tổ chức khóa học "Nâng cao phương pháp } \\
\text { giảng dạy giúp sinh viên học tập chủ động } \\
\text { và trải nghiệm, đạt các chuẩn đầu ra theo } \\
\text { CDIO" chuyên đề } 2 \\
\text { Thành phần: Cán bộ, giảng viên đã đăng ký } \\
\text { theo lịch }\end{array}$ & $\begin{array}{l}\text { PHT - TS. Trần } \\
\text { Đức Thuận }\end{array}$ & G303 & \\
\hline \multirow{2}{*}{$\begin{array}{c}\text { Thứ } 3 \\
\text { 23-08-2016 }\end{array}$} & $07 \mathrm{~h} 30$ & $\begin{array}{l}\text { Tổ chức khóa học "Nâng cao phương pháp } \\
\text { giảng dạy giúp sinh viên học tập chủ động } \\
\text { và trải nghiệm, đạt các chuẩn đầu ra theo } \\
\text { CDIO" chuyên đề } 2 \\
\text { Thành phần: Cán bộ, giảng viên đã đăng ký } \\
\text { theo lịch }\end{array}$ & $\begin{array}{l}\text { PHT - TS. Trần } \\
\text { Đức Thuận }\end{array}$ & $\begin{array}{l}\text { Phòng họp } \\
3\end{array}$ & \\
\hline & $13 \mathrm{~h} 30$ & $\begin{array}{l}\text { Tổ chức khóa học "Nâng cao phương pháp } \\
\text { giảng dạy giúp sinh viên học tập chủ động } \\
\text { và trải nghiệm, đạt các chuẩn đầu ra theo } \\
\text { CDIO" chuyên đề } 2 \\
\text { Thành phần: Cán bộ, giảng viên đã đăng ký } \\
\text { theo lịch }\end{array}$ & $\begin{array}{l}\text { PHT - TS. Trần } \\
\text { Đức Thuận }\end{array}$ & $\begin{array}{l}\text { Phòng họp } \\
3\end{array}$ & \\
\hline \multirow{2}{*}{$\begin{array}{c}\text { Thứ } 2 \\
\text { 24-08-2016 }\end{array}$} & $07 \mathrm{~h} 30$ & $\begin{array}{l}\text { Tổ chức khóa học "Nâng cao phương pháp } \\
\text { giảng dạy giúp sinh viên học tập chủ động } \\
\text { và trải nghiệm, đạt các chuẩn đầu ra theo } \\
\text { CDIO" chuyên đề } 2 \\
\text { Thành phần: Cán bộ, giảng viên đã đăng ký } \\
\text { theo lịch }\end{array}$ & $\begin{array}{l}\text { PHT - TS. Trần } \\
\text { Đức Thuận }\end{array}$ & $\begin{array}{l}\text { Phòng họp } \\
3\end{array}$ & \\
\hline & $13 \mathrm{~h} 30$ & $\begin{array}{l}\text { Tổ chức khóa học "Nâng cao phương pháp } \\
\text { giảng dạy giúp sinh viên học tập chủ động } \\
\text { và trải nghiệm, đạt các chuẩn đầu ra theo } \\
\text { CDIO" chuyên đề } 2 \\
\text { Thành phần: Cán bộ, giảng viên đã đăng ký } \\
\text { theo lịch }\end{array}$ & $\begin{array}{l}\text { PHT - TS. Trần } \\
\text { Đức Thuận }\end{array}$ & $\begin{array}{l}\text { Phòng họp } \\
3\end{array}$ & \\
\hline $\begin{array}{c}\text { Thứ } 5 \\
25-08-2016\end{array}$ & 07h30 & $\begin{array}{l}\text { Tổ chức khóa học "Nâng cao phương pháp } \\
\text { giảng dạy giúp sinh viên học tập chủ động } \\
\text { và trải nghiệm, đạt các chuẩn đầu ra theo } \\
\text { CDIO" chuyên đề } 2\end{array}$ & $\begin{array}{l}\text { PHT - TS. Trần } \\
\text { Đức Thuận }\end{array}$ & A201 & \\
\hline
\end{tabular}




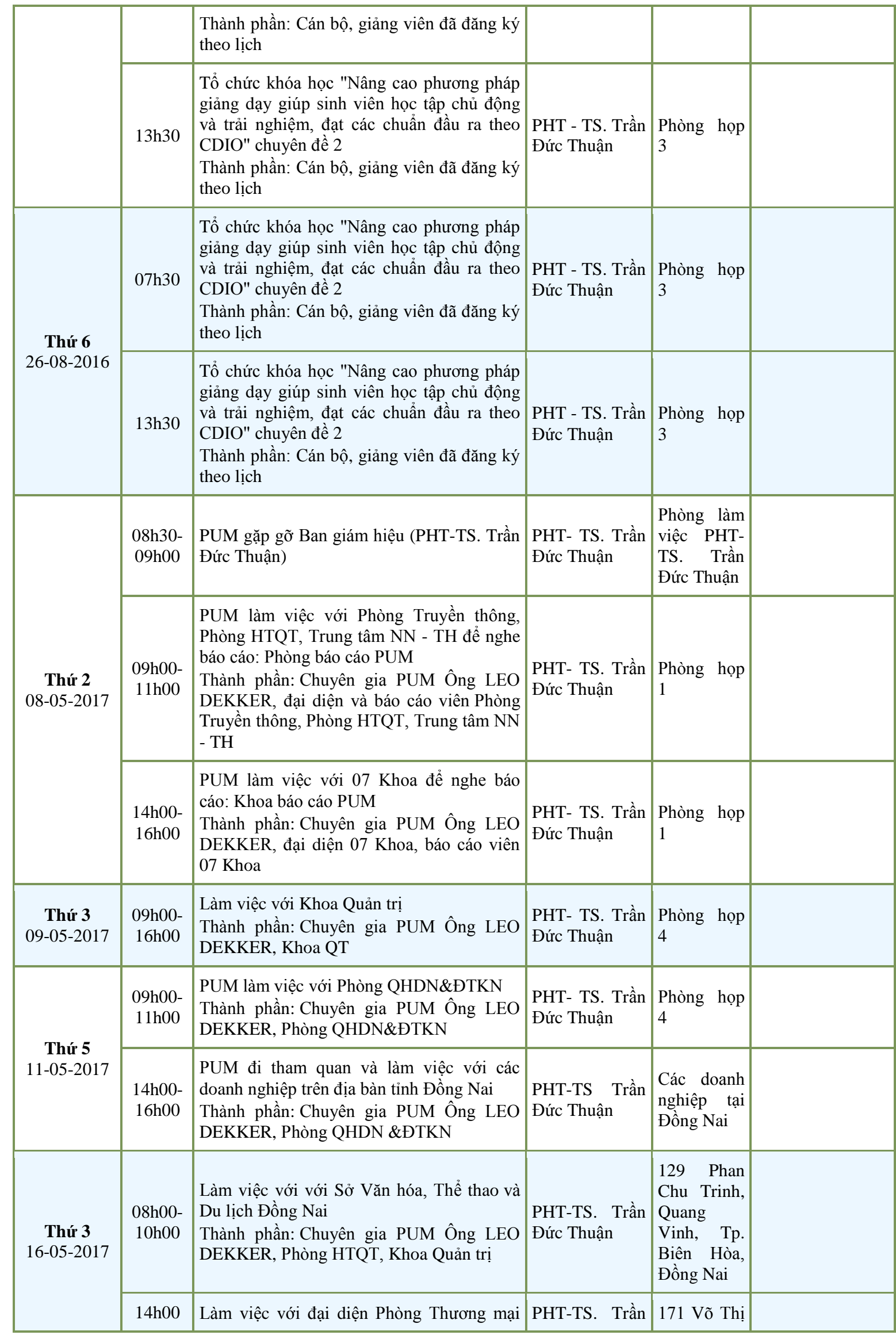




\begin{tabular}{|c|c|c|c|c|}
\hline & & $\begin{array}{l}\text { và Công nghiệp Việt Nam (VCCI) về lĩnh } \\
\text { vực Nhà hàng - Khách sạn } \\
\text { Thành phần: Chuyên gia PUM Ông LEO } \\
\text { DEKKER, Phòng HTQT, Khoa Quản trị }\end{array}$ & Đức Thuận & $\begin{array}{l}\text { Sáu, P.7, Q. } \\
\text { 3, TP.HCM }\end{array}$ \\
\hline $\begin{array}{c}\text { Thứ } 4 \\
17-05-2016\end{array}$ & $15 \mathrm{~h} 00$ & $\begin{array}{l}\text { Gặp gỡ tập đoàn Khách sạn IHG } \\
\text { (InterContinental Hotels Group) tại Việt } \\
\text { Nam } \\
\text { Thành phần: Chuyên gia PUM Ông LEO } \\
\text { DEKKER, Phòng HTQT, Khoa Quản trị }\end{array}$ & $\begin{array}{l}\text { PHT-TS. Trần } \\
\text { Đức Thuận }\end{array}$ & $\begin{array}{l}\text { Tòa nhà } \\
\text { INTERCO } \\
\text { NTINENT } \\
\text { AL } \\
\text { SAIGON, } \\
\text { Đường Lê } \\
\text { Duẩn, Q1, } \\
\text { Tp.HCM }\end{array}$ \\
\hline $\begin{array}{l}\text { Thứ } 6 \\
19-05-2017\end{array}$ & $\begin{array}{l}09 \mathrm{~h} 00- \\
11 \mathrm{~h} 00\end{array}$ & $\begin{array}{l}\text { PUM báo cáo tổng kết nhiệm vụ số } 07 \text {, Báo } \\
\text { cáo viên: chuyên gia LEO DEKKER } \\
\text { Thành phần: Chuyên gia LEO } \\
\text { DEKKER, Trường, Phó Phòng: P.Truyền } \\
\text { thông, Phòng HTQT, Phòng QHDN \& } \\
\text { ĐTKN; Trưởng, Phó Trung tâm NN - TH; } \\
\text { Trưởng, Phó } 07 \text { Khoa }\end{array}$ & $\begin{array}{l}\text { PHT- TS. Trần } \\
\text { Đức Thuận }\end{array}$ & $\begin{array}{l}\text { Phòng họp } \\
1\end{array}$ \\
\hline $\begin{array}{c}\text { Thứ } 3 \\
13-03-2018\end{array}$ & $\begin{array}{l}09 \mathrm{~h} 00- \\
11 \mathrm{~h} 00\end{array}$ & $\begin{array}{l}\text { Làm việc với chuyên gia PUM } \\
\text { Thành phần: Phòng HTQT }\end{array}$ & $\begin{array}{l}\text { PHT- TS. Trần } \\
\text { Đức Thuận }\end{array}$ & $\begin{array}{l}\text { Phòng họp } \\
2\end{array}$ \\
\hline
\end{tabular}

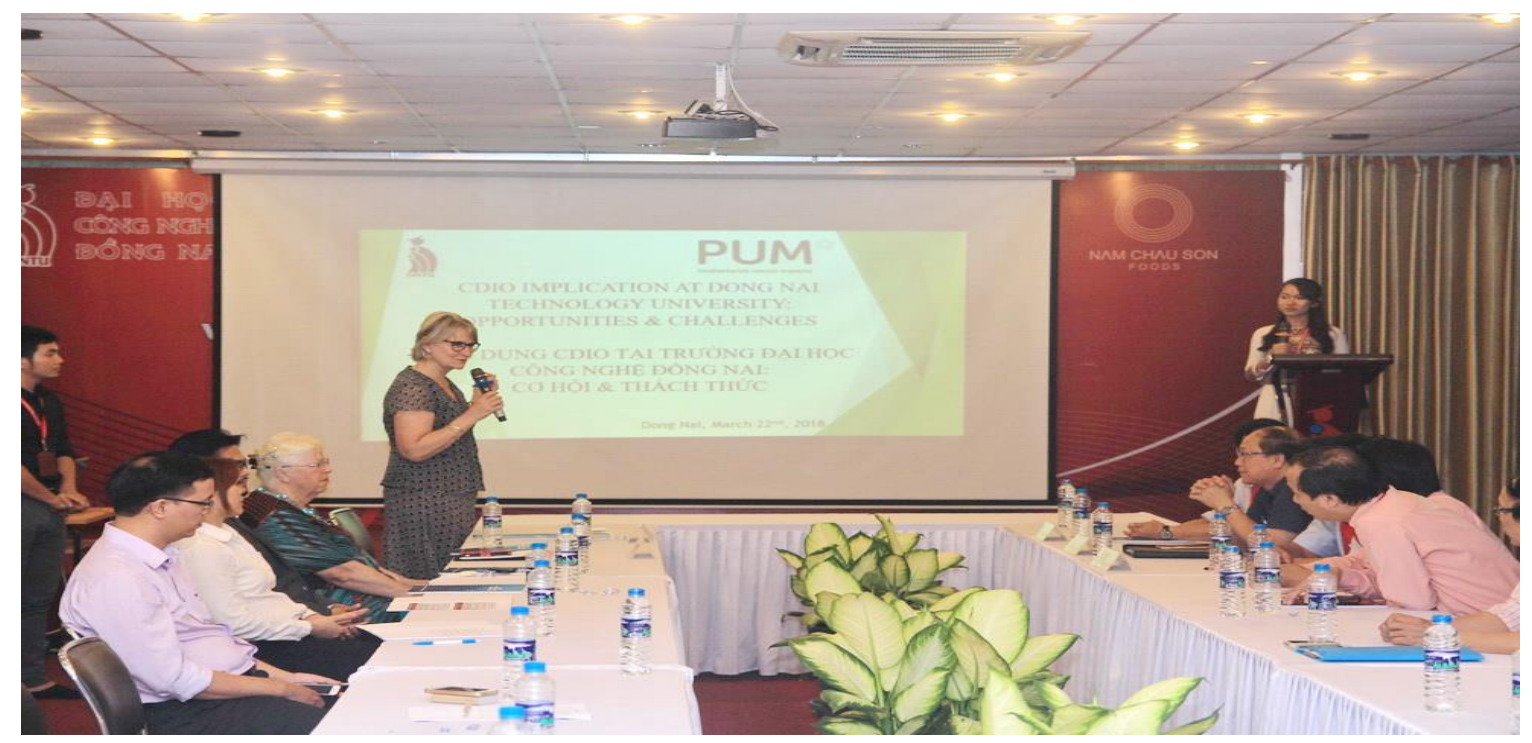

Hinh 1. Buổi tổng kết về hoạt động hợp tác với tổ chức PUM trong việc úng dụng CDIO taii DNTU 


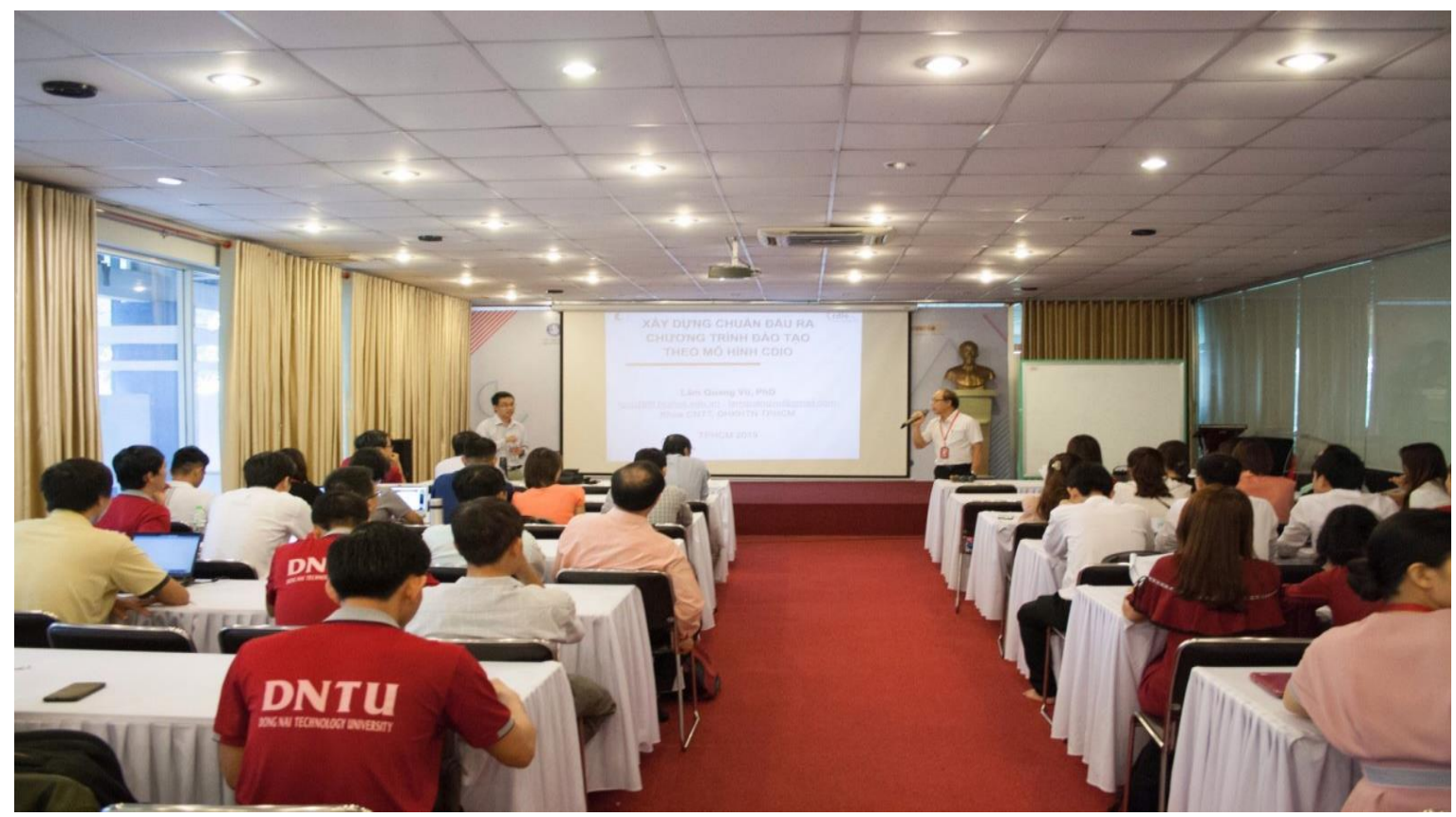

Hình 2. Tập huấn xây dụng chuẩn đầu ra chưong trình đào tạo theo mô hình CDIO

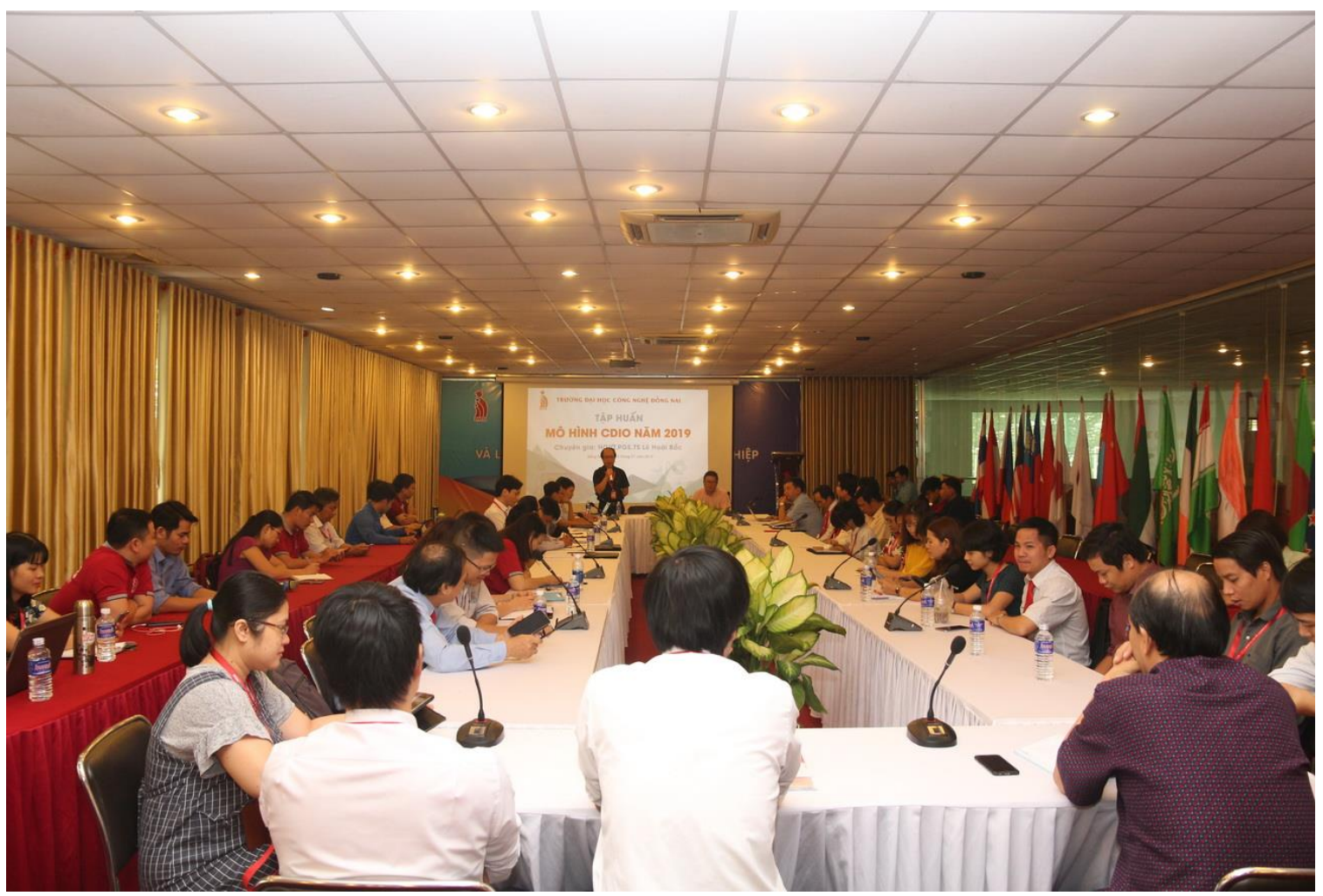

Hinh 3. Tập huấn Mô hình CDIO 
